\title{
Signet-ring Cell Carcinoma Derived from a Main Duct-type Intraductal Papillary Mucinous Neoplasm of the Pancreas: A Case Report with Long-term Follow-up
}

\author{
Toshitaka Sakai ${ }^{1}$, Shinsuke Koshita ${ }^{1}$, Kei Ito ${ }^{1}$, Yoshihide Kanno ${ }^{1}$, Takahisa Ogawa ${ }^{1}$, \\ Hiroaki Kusunose ${ }^{1}$, Kaori Masu ${ }^{1}$, Yujiro Kawakami ${ }^{1}$, Yuki Fujii ${ }^{1}$, Touji Murabayashi ${ }^{1}$, \\ Sho Hasegawa ${ }^{1}$, Fumisato Kozakai ${ }^{1}$, Yutaka Noda ${ }^{1}$, Masaya Oikawa ${ }^{2}$, \\ Takashi Tsuchiya $^{2}$ and Takashi Sawai ${ }^{3}$
}

\begin{abstract}
:
We herein report the case of a 74-year-old man who underwent surgery 9 years after his initial visit and who was pathologically diagnosed with signet-ring cell carcinoma (SRCC) derived from a main-duct-type intraductal papillary mucinous neoplasm (MD-IPMN). At the first imaging examination, only a small pancreatic cyst with mild dilation of the main pancreatic duct (MPD) was detected in the pancreatic head. Eventually, MD-IPMN with mural nodules and MPD dilation $(30 \mathrm{~mm})$ developed in the pancreatic body, while the pancreatic head cyst remained unchanged. Total pancreatectomy was performed and the MD-IPMN was pathologically diagnosed as SRCC derived from an intestinal-type MD-IPMN.
\end{abstract}

Key words: IPMN, signet-ring cell carcinoma, intestinal type, mass forming pancreatitis

(Intern Med 57: 1093-1099, 2018)

(DOI: 10.2169/internalmedicine.9743-17)

\section{Introduction}

Intraductal papillary mucinous neoplasms (IPMNs) are mucin-producing neoplasms that originate in the pancreatic ducts. IPMNs are classified into three types: main-duct IPMN (MD-IPMN), branch-duct IPMN (BD-IPMN), and mixed-type IPMN, based on imaging studies and the histology. MD-IPMNs are characterized by the segmental or diffuse dilation of the main pancreatic duct (MPD) to $>5 \mathrm{~mm}$ without any other causes of MPD obstruction (1). The incidence of malignancy (including high-grade dysplasia) and invasive IPMNs in patients with MD-IPMNs is reported to be $61.6 \%$ and $43.1 \%$, respectively. Considering these high rates of malignancy, surgical resection is strongly recommended for all surgically-fit patients $(1,2)$; thus, there are few studies on the predictors of malignancy in MD-IPMNs and few studies involving the long-term follow-up of pa- tients with MD-IPMNs $(3,4)$. In addition, most cases of pancreatic ductal carcinoma derived from IPMNs are histologically diagnosed as tubular adenocarcinoma or mucinous adenocarcinoma (5); other histological types are rare. We herein report a case of signet-ring cell carcinoma that developed from an MD-IPMN after 9 years of follow-up.

\section{Case Report}

A pancreatic cystic lesion was identified in a 74-year-old man by ultrasonography; the patient was referred to our hospital to undergo a detailed examination in 2006. Endoscopic ultrasonography (EUS) showed a cystic lesion of $15 \mathrm{~mm}$ in diameter in the pancreatic head and dilation of the MPD to $5 \mathrm{~mm}$, resulting in the diagnosis of BD-IPMN and a followup strategy based on regular imaging examinations was implemented. In 2007, magnetic resonance cholangiopancreatography (MRCP) demonstrated that the diameter of the

${ }^{1}$ Department of Gastroenterology, Sendai City Medical Center, Japan, ${ }^{2}$ Department of Surgery, Sendai City Medical Center, Japan and ${ }^{3}$ Department of Pathology, Sendai City Medical Center, Japan

Received: July 1, 2017; Accepted: August 17, 2017; Advance Publication by J-STAGE: December 21, 2017

Correspondence to Dr. Toshitaka Sakai, t.sakai@openhp.or.jp 


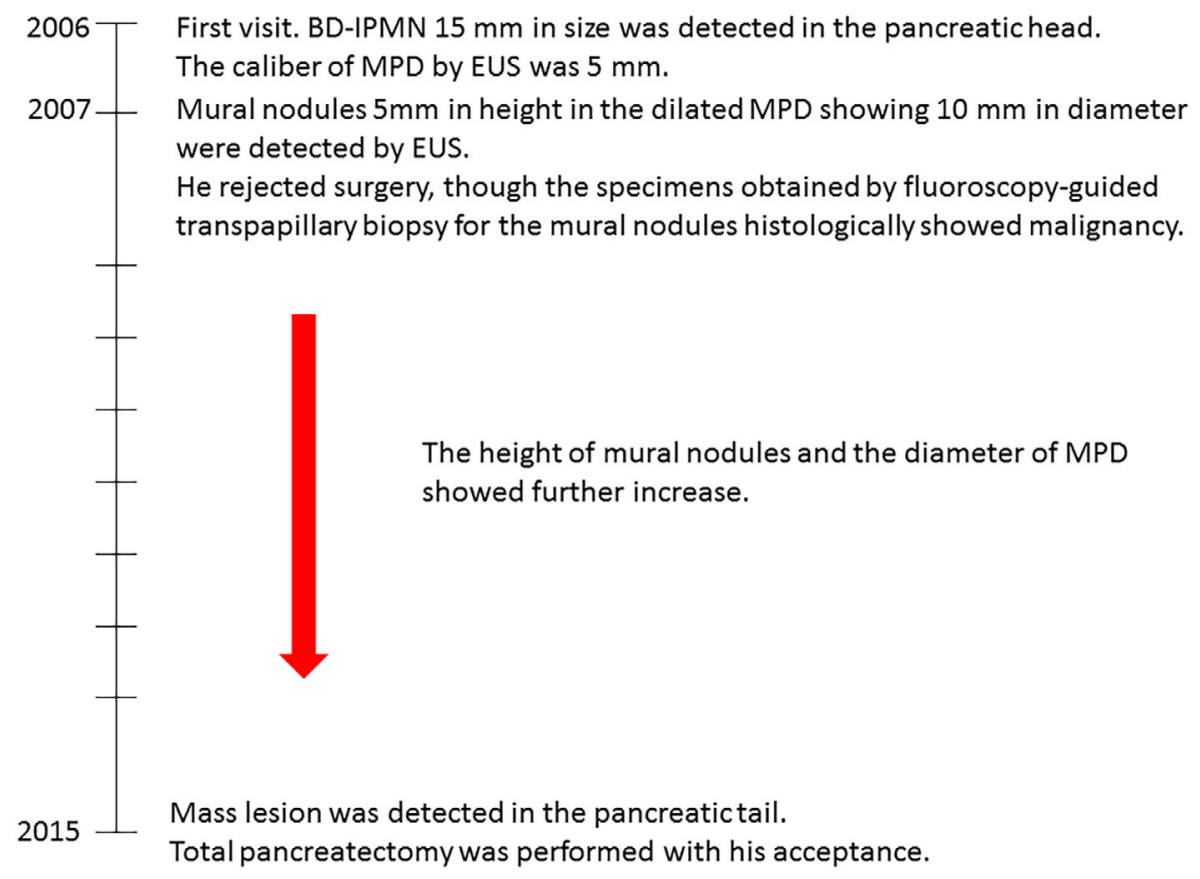

Figure 1. The clinical course of this patient.
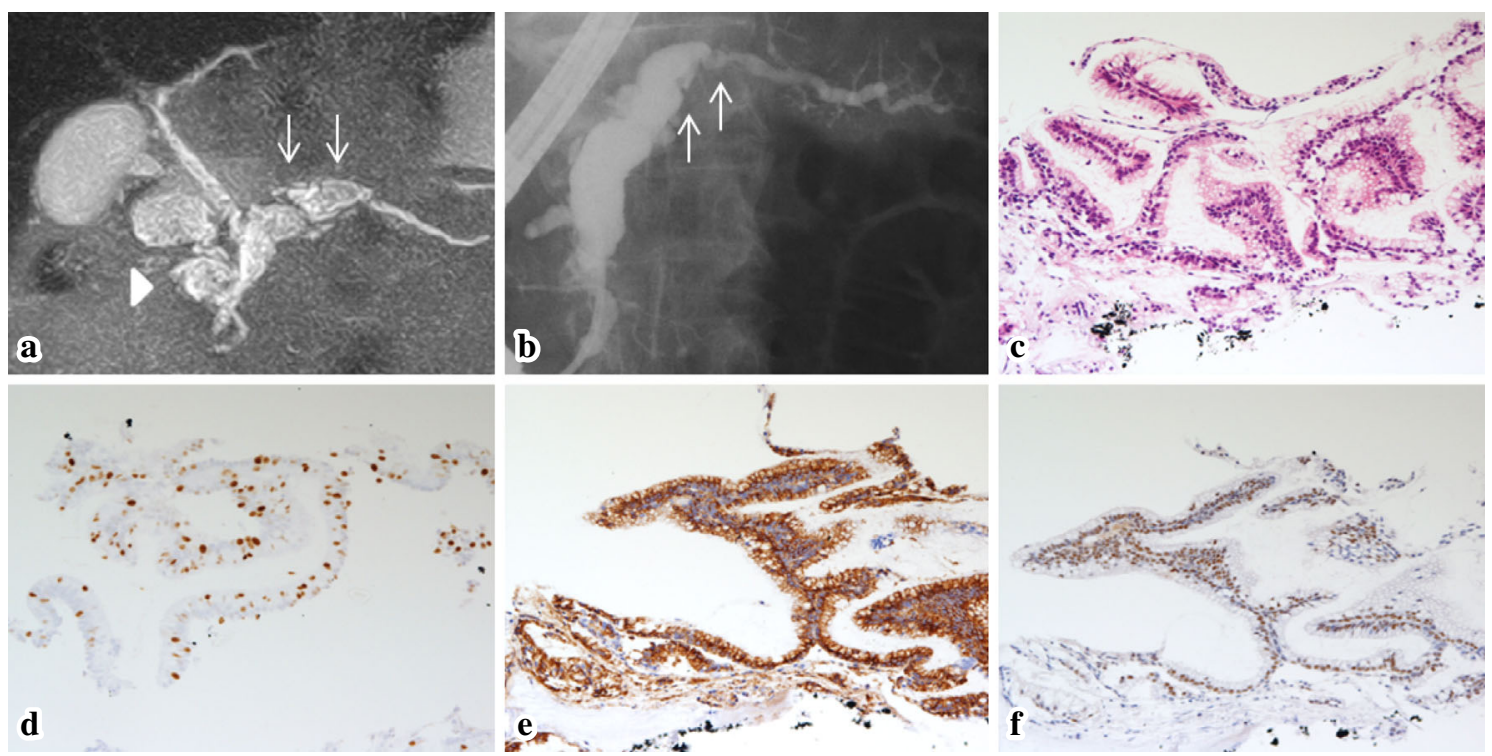

Figure 2. (a) MRCP (in 2007): The MPD in the pancreatic body was dilated to $10 \mathrm{~mm}$ (arrow), whereas the size of the pancreatic cyst in the pancreatic head remained unchanged (arrowhead). (b) ERP (in 2008) was performed to investigate the suspected mural nodules, which showed filling defects in the dilated MPD (arrow). Fluoroscopy-guided transpapillary biopsy was performed. (c-f) The histological findings of the biopsy specimens. Hematoxylin and Eosin staining (c, orig. mag. $\times 50)$ showed that the papillary epithelium was composed of atypical cells with mucus. The Ki67 labeling index (d, orig. mag. $\times 50)$ was $30 \%$, and the specimen was diffusely positive for both MUC2 (e, orig. mag. $\times 50)$ and CDX2 (f, orig. mag. $\times 50$ ). MRCP: magnetic resonance cholangiopancreatography, MPD: main pancreatic duct, ERP: endoscopic retrograde pancreatography

MPD was $10 \mathrm{~mm}$ (Fig. 2a), and EUS showed a mural nodule of $5 \mathrm{~mm}$ in height in the MPD; the cystic lesion in the pancreatic head was unchanged. These findings suggested the development of MD-IPMN in the pancreatic body. Endoscopic retrograde pancreatography (ERP) showed filling defects, suggesting mucins or mural nodules in the dilated MPD. Intraductal ultrasonography revealed that some of these filling defects in the pancreatic body were mural nodules, and fluoroscopy-guided transpapillary biopsy was performed to obtain a sample of the mural nodules (Fig. 2b). 


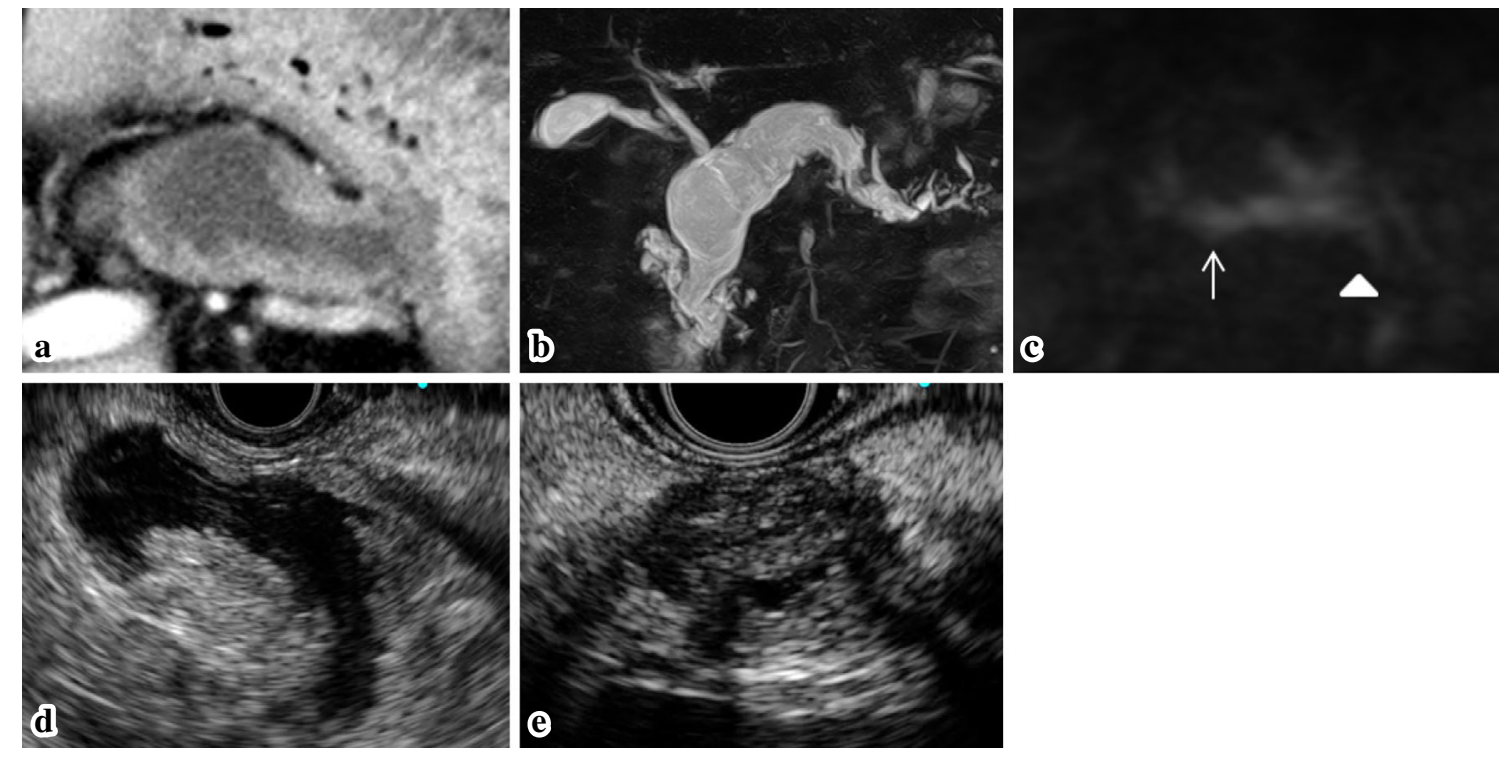

Figure 3. The imaging findings in 2015. (a) CECT: Mural nodules were detected in the dilated MPD of the pancreatic body. (b) MRCP: The MPD in the pancreatic body was dilated to $30 \mathrm{~mm}$, whereas the pancreatic cyst in the pancreatic head was the same size as in 2007. (c) MRI-DWI: Positive signals were detected in the pancreatic body (arrow) and tail (arrowhead). EUS: Mural nodules of $12 \mathrm{~mm}$ in height in the MPD of the pancreatic body (d), and a well-circumscribed, low echoic mass lesion of $15 \mathrm{~mm}$ in diameter was detected in the pancreatic tail (e). CECT: contrast enhanced computed tomography, MPD: main pancreatic duct, MRCP: magnetic resonance cholangiopancreatography, DWI: diffusion weight imaging, EUS: endoscopic ultra sonography

Histologically, the specimens showed a papillary epithelium that was composed of atypical cells with stratified nuclei [identified by hematoxylin and eosin (HE) staining]; the Ki 67 labeling index (LI) was high (30\%). In addition, the specimen was diffusely positive for both mucin 2 glycoprotein (MUC2) and caudal-type homeobox 2 (CDX2) (Fig. 2c-f). Although the histological findings of the specimens suggested a malignant intestinal-type IPMN, the patient rejected surgery. During the subsequent follow-up period, the height of mural nodule and the diameter of MPD showed further increases. Nevertheless, we could not persuade him to undergo pancreatic surgery. In 2015, EUS revealed a low echoic mass lesion of $15 \mathrm{~mm}$ in size in the pancreatic tail, suggesting the development of pancreatic carcinoma concomitant with IPMN, and the patient was admitted to our medical center for a detailed examination.

A laboratory analysis revealed that the patient's serum HbA1c level was high (8.0\%), while his serum carcinoembryonic antigen (CEA) level was within the normal range (3.7 ng/mL), whereas his serum carbohydrate antigen 19-9 (CA19-9) level was elevated (111.6 U/mL). Contrast enhanced computed tomography (CECT) showed a cystic lesion of $15 \mathrm{~mm}$ in size (suggesting BD-IPMN) in the pancreatic head and the dilation of the MPD to $30 \mathrm{~mm}$, mainly in the pancreatic body, in which multiple mural nodules were detected (Fig. 3a). The suspected pancreatic mass lesion in the pancreatic tail that had been detected by EUS was unclear. Magnetic resonance imaging (MRI) also showed a cystic lesion in the pancreatic head and MPD dilation, mainly in the pancreatic body (Fig. 3b). Diffusion weight imaging (DWI) showed positive signals in the pancreatic body and tail (Fig. 3c). EUS showed mural nodules of 12 $\mathrm{mm}$ in height in the MPD of the pancreatic body (Fig. 3d). In addition, a well-circumscribed, low echoic mass lesion of $15 \mathrm{~mm}$ in diameter was detected in the pancreatic tail and pancreatic carcinoma concomitant with IPMN was suspected (Fig. 3e). Based on these findings, our preoperative diagnosis was malignant MD-IPMN and pancreatic carcinoma concomitant with the IPMN in the pancreatic tail. The Total pancreatectomy was performed with the patient's consent. The resected specimen macroscopically showed papillary tumors filling the remarkably dilated MPD of the pancreatic body and a white pancreatic parenchyma (suggestive of fibrotic change) in the pancreatic tail. Histologically, a papillary epithelium composed of atypical cells showing stratified nuclei was detected, mainly in the pancreatic body. The histological analysis of a sample of the epithelium revealed a Ki67 LI of 30\%, with focal p53 positivity. The specimen was diffusely positive for both MUC2 and CDX2. These findings contributed to a diagnosis of malignant intestinaltype IPMN (Fig. 4a-d). In addition, signet-ring cell carcinoma (SRCC) was detected under the malignant epithelium and was positive for both MUC2 and CDX2 (Fig. 4e-h). Although the histological transition the malignant IPMN and SRCC was unclear, the SRCC was considered to have been derived from the IPMN of the epithelium due to the similarity of the immunohistological findings. SRCC was also detected in the lymph nodes adjacent to the pancreatic body. 


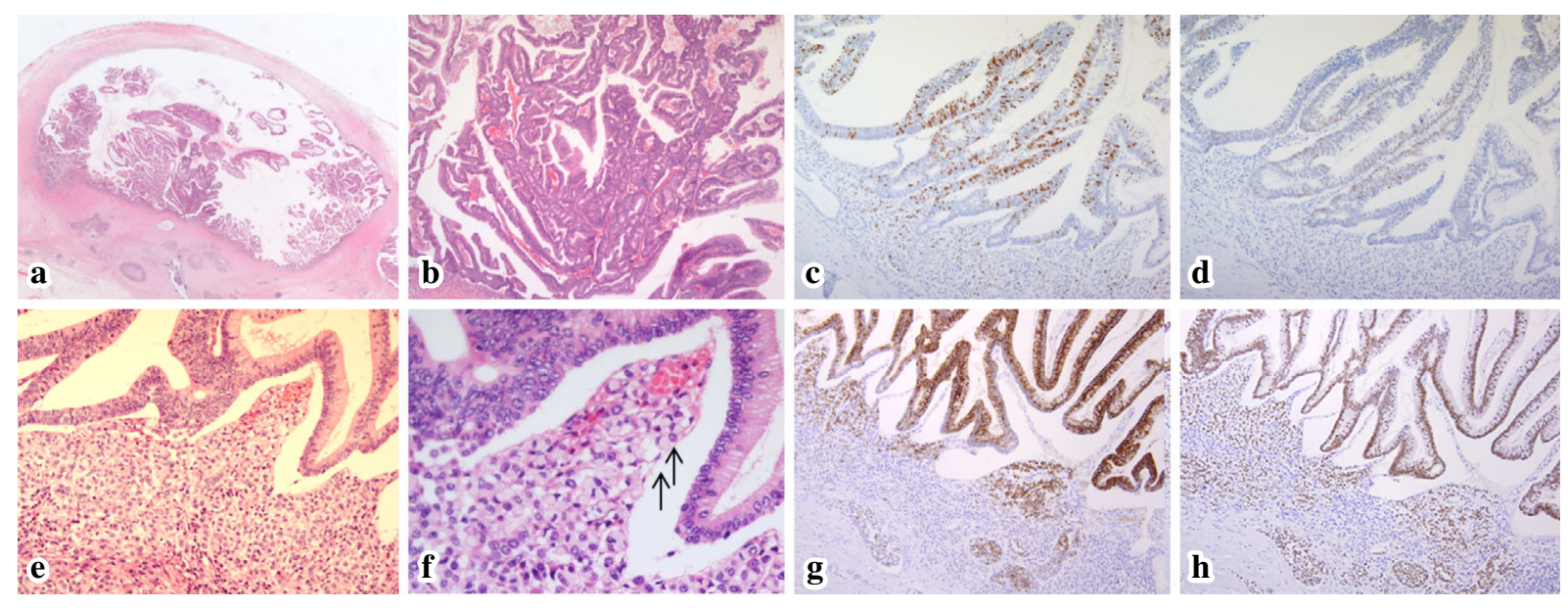

Figure 4. The histological findings of the pancreatic body of the resected specimen. Hematoxylin and Eosin (H\&E) staining showed a papillary epithelium in the dilated MPD (a, orig. mag. $\times 1.25)$. The epithelium was composed of atypical cells with stratified nuclei $(b$, orig. mag. $\times 50)$. TheKi67 LI of this epithelium (c) was $30 \%$, and p53 staining (d) was positive, which revealed malignancy. In addition, H\&E staining showed the diffuse distribution of SRCC under the malignant papillary epithelium (arrow) (e, orig. mag. $\times 50$; f, orig. mag. $\times 100$ ), and both the SRCC and the malignant epithelium were positive for MUC2 (g, orig. mag. $\times 50)$ and $\operatorname{CDX} 2(\mathrm{~h}$, orig. mag. $\times 50)$, which was consistent with intestinal-type IPMN. MPD: main pancreatic duct, SRCC: signet-ring cell carcinoma, MUC2: mucin 2 glycoprotein, CDX2: caudal-type homeobox 2, IPMN: intraductal papillary mucinous neoplasm
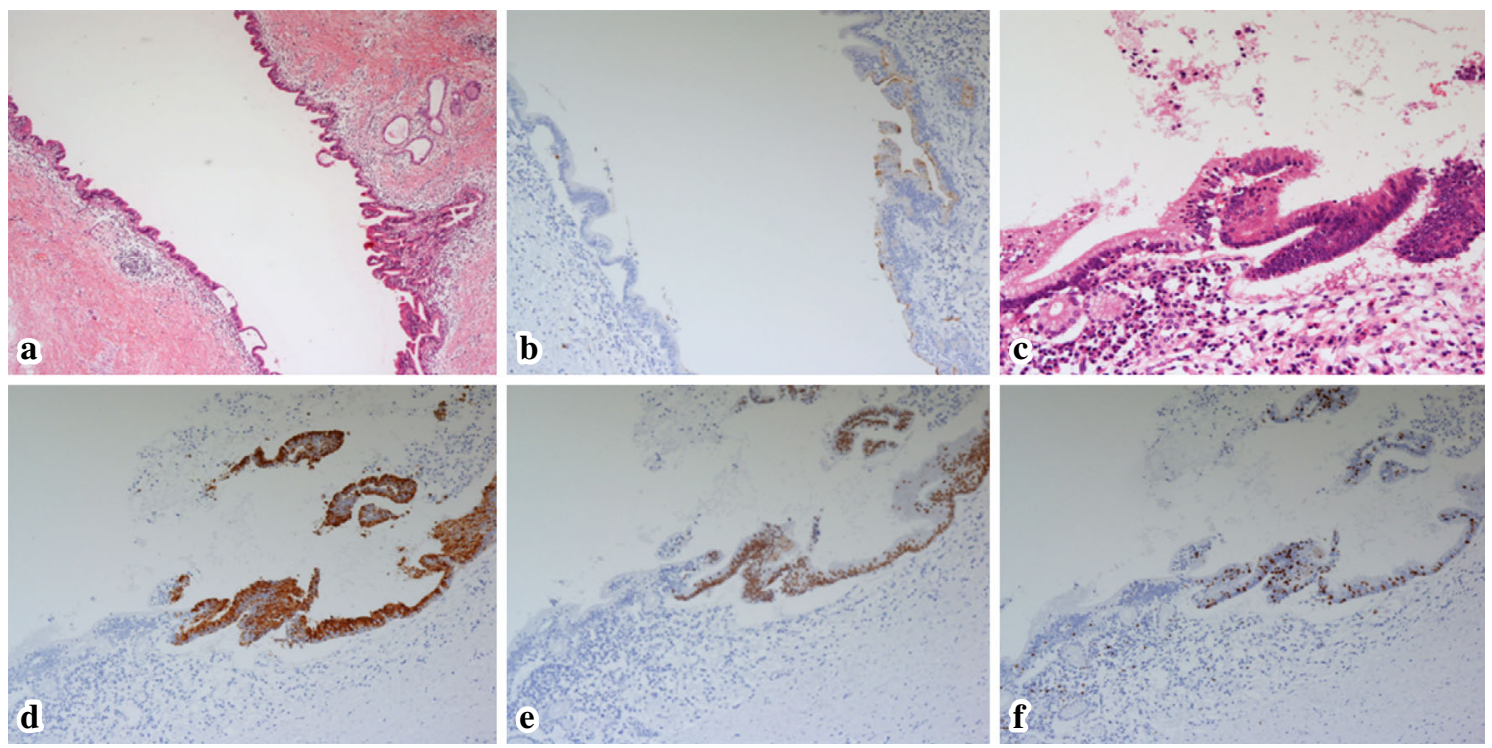

Figure 5. The histological findings of a cystic lesion in a resected specimen of the pancreatic head. Hematoxylin and Eosin $($ H\&E) staining (a, orig. mag. $\times 25)$ demonstrated low papillary epithelium in this cystic lesion. The epithelium was focally positive for MUC1 (b, orig. mag. $\times 25)$ and negative for MUC2 and CDX2, indicating gastric-type IPMN. Additionally, in the marginal position of this cyst, there was a clear border between the gastric-type IPMN and the intestinal-type IPMN (similar to the lesion of the pancreatic body) (c, H\&E staining, orig. mag. $\times 50$; d, MUC2 staining, orig. mag. $\times 25$; e, CDX2 staining, orig. mag. $\times 25$; f, Ki67 staining, orig. mag. $\times 25)$. MUC2: mucin 2 glycoprotein, MUC1: mucin 1 glycoprotein, CDX2: caudal-type homeobox 2, IPMN: intraductal papillary mucinous neoplasm

With regard to the pancreatic cyst in the pancreatic head, a histological examination of the epithelium revealed findings that were similar to pyloric glandular epithelium. An immunohistochemical analysis of the epithelium revealed a low Ki67 LI, p53 negativity, focal MUC1 positivity, MUC2 negativity, diffuse MUC5AC positivity, focal MUC6 positivity, and CDX2 negativity, resulting in a diagnosis of gastrictype IPMN with low-grade dysplasia (Fig. 5a, b). In the pancreatic neck, there was a clear border between the epithelium of the gastric-type IPMN and that of the intestinal- 


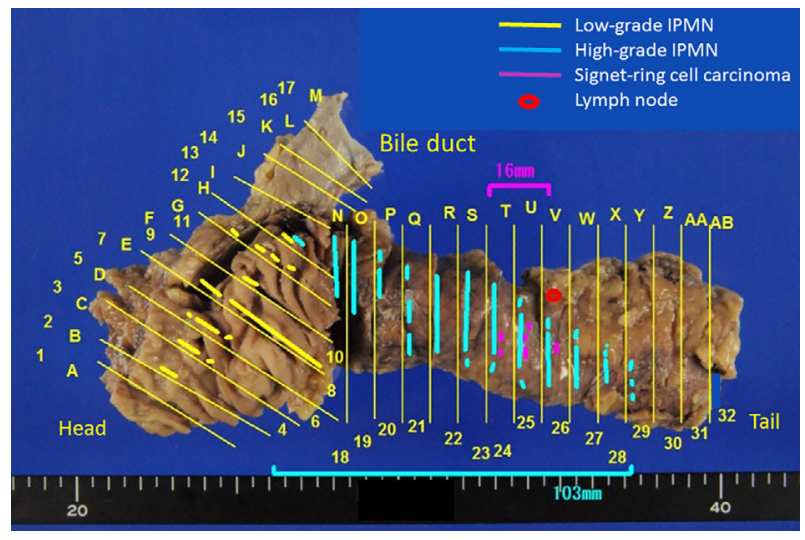

Figure 6. The mapping of the resected specimens. Histologically, an IPMN with low-grade dysplasia of the gastric type was located in the pancreatic head, whereas intestinal-type IPMN with high-grade dysplasia was located over a length of $10 \mathrm{~cm}$ from the pancreatic body to tail. A 16-mm lesion of signet-ring cell carcinoma was located adjacent to the high-grade IPMN. IPMN: intraductal papillary mucinous neoplasm

type IPMN; the border was considered to be a transitional position or a collisional position (Fig. 5c-f, 6). With regard to the pancreatic mass lesion in the pancreatic tail, fibrosis and the infiltration of inflammatory cells were detected, but infiltration of IgG4-positive cells was not detected. The mass lesion was pathologically diagnosed as mass-forming chronic pancreatitis. The TNM classification was IIB (T2N1 M0). Recurrence has not been detected in one year since the operation.

\section{Discussion}

According to the International Consensus Guidelines (revised in 2012) on the management of IPMN and MCN of the pancreas, MD-IPMNs are considered to be an indication for surgery because of their high frequency of malignancy and the low survival rate of afflicted subjects. In these guidelines, MPD dilation of 5-9 mm was considered to be "worrisome feature", as is the case of BD-IPMNs. A few reports have been published on the indications for surgery or predictors of malignancy in patients with MD-IPMNs. One of these reports notes that the dilation of the MPD to a diameter of $\geq 5 \mathrm{~mm}$ is a risk factor of malignancy, whereas another report described that conservative follow-up in patients with MD-IPMNs with a MPD diameter of $<15 \mathrm{~mm}$, negative cytology or no mural nodules may be acceptable $(3,4,6)$. Generally, however, the diagnosis of MDIPMN is regarded as an indication for surgery due to the lack of adequate predictive factors for malignancy in MDIPMNs. As for the present case, surgery was indicated in 2007 when malignancy was pathologically diagnosed following a transpapillary biopsy of the mural nodules. If this patient had agreed to surgery at that time, the development into invasive carcinoma could possibly have been prevented. Because it has been reported that IPMNs have a worse prog- nosis if they involve massively invasive carcinoma $(5,7)$, this patient should have undergone surgery at an earlier stage. In this case, it was difficult to detect the transformation to invasive carcinoma based on imaging studies before surgery; however the possible involvement of invasive carcinoma was suggested by the increased diameter of the MPD and the increased height of the mural nodules. However, the pancreatic parenchyma in the pancreatic body and tail showed positive signals on MRI-DWI, which can imply invasive cancer. MRI-DWI is reportedly useful for the diagnosis of malignancy in patients with IPMNs (8); thus, it may also contribute to detecting the development of invasive cancer during follow-up in patients with IPMN.

Furukawa et al. proposed the morphological classification of IPMNs into four pathological subtypes, namely, the gastric, intestinal, pancreatobiliary and oncocytic types based on the histomorphological features of the papillae and the immunohistochemical features of mucin glycoproteins (9). In the present case, the IPMN in the pancreatic body was pathologically diagnosed as intestinal-type due to the expression of both MUC2 and CDX2. With regard to the general characteristics of intestinal-type IPMNs, it is reported that $80 \%$ of intestinal-type IPMNs are malignant and that the frequency of the intestinal type among MD-IPMNs is high. However, the prognosis of invasive carcinoma derived from intestinal-type IPMN is reported to be better in comparison to the other subtypes (10-12). This prognostic difference may originate in differences in the histological types of the invasive lesions because some invasive carcinomas derived from intestinal-type IPMNs are mucinous adenocarcinoma, whereas all invasive carcinoma derived from IPMNs of other pathological subtypes are tubular adenocarcinoma (11). On the other hand, this patient, whose pancreatic body IPMN was pathologically diagnosed as the intestinal type, surprisingly developed SRCC, which metastasized to a lymph node. It is reported that tubular adenocarcinoma and mucinous adenocarcinoma account for $>98 \%$ of invasive carcinoma derived from IPMNs (5); thus, other histological types of invasive carcinoma are rare. We could only find one case report of SRCC derived from an IPMN (13). The case involved mixed mucinous adenocarcinoma and SRCC, while the invasive carcinoma of the present patient consisted solely of SRCC, which indicates the value of the present case. In addition, the pathological subtype of the IPMN in the above-mentioned case report was likely the intestinal type (the same as the present case). In the literature, most cases of SRCC are reported to develop in the stomach, while some cases develop in the colon, lung, breast, thyroid, and other sites. It is reported that the mucin expression patterns of SRCC differ according to the location $(14,15)$. SRCCs of the stomach or breast are MUC1-positive, while SRCCs of the colon are MUC2-positive and MUC1negative. Thus, it can be said that the immunostaining pattern of SRCC in this case was similar to the pattern observed in SRCC of the colon.

In this case, the pathological examination of the BD- 
IPMN of in the pancreatic head, which was approximately $15 \mathrm{~mm}$ in diameter and which had not changed since 2006, revealed low-grade dysplasia and the gastric type. Namely, two IPMNs of different pathological subtypes existed within the same pancreas. Histological and immunohistochemical examinations revealed that these two adjacent IPMNs formed a clear border. Two possible mechanisms for the development of IPMNs in cases in which both gastric and intestinal-type IPMN coexist have been reported: one is the possibility of a morphological change from gastric-type to intestinal-type; and the other is the possibility of the collision between the two types of IPMNs after they develop separately $(16,17)$. As with those reports, although it is difficult to clarify the relationship between gastric- and intestinal-type IPMNs, it is interesting that the IPMNs in the present case followed completely different clinical courses. This suggests that the pathological subtypes of IPMNs strongly affect their natural histories (18).

In this patient, mass forming pancreatitis in the pancreatic tail, which existed separately from the MD-IPMN in the pancreatic body, was also detected. Some reports have described the development of autoimmune pancreatitis (AIP) around IPMNs $(19,20)$, whereas a literature search revealed no cases of IPMN involving mass forming pancreatitis without AIP. Considering that some cases of IPMN develop acute pancreatitis due to obstruction of the MPD by mucin production, a mechanism like this might have brought about the change of chronic pancreatitis in the present case, followed by the development of mass-forming pancreatitis. Although the performance of EUS-FNA was considered in order to clarify the diagnosis of the mass lesion, it was ultimately not performed (with the patient's agreement) because we were of the opinion that the pathological diagnosis of the mass lesion would not affect the therapeutic strategy, as total pancreatectomy was required as radical surgery for malignant IPMN. In addition to pancreatic carcinoma concomitant with IPMN mass forming pancreatitis (including localized autoimmune pancreatitis) may develop during the follow-up of patients with IPMNs; thus, these diseases should be discriminated when a mass lesion is detected in patients with IPMNs.

In conclusion, we experienced a case of SRCC derived from an MD-IPMN after 9 years of follow-up. Although it is extremely rare, intestinal-type IPMN can progress to SRCC, which is considered to be a relatively aggressive invasive cancer.

The authors state that they have no Conflict of Interest (COI).

\section{Acknowledgement}

We would like to express our appreciation to Dr. Toru Furukawa of Tohoku University, whose comments on our pathological diagnosis of the resected specimens helped to clarify the diagnosis. In addition, we would like to thank the staff of the Department of Pathology at Sendai City Medical Center for performing immunostaining of the resected specimens of this pa- tient.

\section{References}

1. Tanaka M, Fernández-Del Castillo $\mathrm{C}$, Adsay V, et al. International consensus guidelines 2012 for the management of IPMN and MCN of the pancreas. Pancreatology 12: 183-197, 2012.

2. Salvia R, Fernández-del Castillo C, Bassi C, et al. Main-duct intraductal papillary mucinous neoplasms of the pancreas: clinical predictors of malignancy and long-term survival following resection. Ann Surg 239: 678-685, 2004.

3. Ogura T, Masuda D, Kurisu Y, et al. Potential predictors of disease progression for main-duct intraductal papillary mucinous neoplasms of the pancreas. J Gastroenterol Hepatol 28: 1782-1786, 2013.

4. Takuma K, Kamisawa T, Anjiki H, et al. Predictors of malignancy and natural history of main-duct intraductal papillary mucinous neoplasms of the pancreas. Pancreas 40: 371-375, 2011.

5. Yamaguchi K, Kanemitsu S, Hatori T, et al. Pancreatic ductal adenocarcinoma derived from IPMN and pancreatic ductal adenocarcinoma concomitant with IPMN. Pancreas 40: 571-580, 2011.

6. Hackert T, Fritz S, Klauss M, et al. Main-duct intraductal papillary mucinous neoplasm: high cancer risk in duct diameter of 5 to 9 mm. Ann Surg 262: 875-881, 2015.

7. Nara S, Shimada K, Kosuge T, Kanai Y, Hiraoka N. Minimally invasive intraductal papillary-mucinous carcinoma of the pancreas: clinicopathologic study of 104 intraductal papillary-mucinous neoplasms. Am J Surg Pathol 32: 243-255, 2008.

8. Ogawa T, Horaguchi J, Fujita N, et al. Diffusion-weighted magnetic resonance imaging for evaluating the histological degree of malignancy in patients with intraductal papillary mucinous neoplasm. J Hepatobiliary Pancreat Sci 21: 801-808, 2014.

9. Furukawa T, Klöppel G, Volkan Adsay N, et al. Classification of types of intraductal papillary-mucinous neoplasm of the pancreas: a consensus study. Virchows Archiv 447: 794-799, 2005.

10. Mino-Kenudson M, Fernández-del Castillo $\mathrm{C}$, Baba Y, et al. Prognosis of invasive intraductal papillary mucinous neoplasm depends on histological and precursor epithelial subtypes. Gut 60: 17121720, 2011.

11. Sadakari Y, Ohuchida K, Nakata K, et al. Invasive carcinoma derived from the nonintestinal type intraductal papillary mucinous neoplasm of the pancreas has a poorer prognosis than that derived from the intestinal type. Surgery 147: 812-817, 2010.

12. Distler M, Kersting S, Niedergethmann M, et al. Pathohistological subtype predicts survival in patients with intraductal papillary mucinous neoplasm (IPMN) of the pancreas. Ann Surg 258: 324-330, 2013.

13. Naito $Y$, Okabe Y, Morimitsu $Y$, et al. A case of invasive carcinoma derived from intraductal papillary-mucinous carcinoma of the pancreas producing a signet ring-type appearance on pancreatic duct brushing cytology. Cytopathology 20: 406-408, 2009.

14. Chu PG, Weiss LM. Anatomic pathology/signet-ring cell carcinomas of the stomach, breast, and colon immunohistochemical characterization of signet-ring cell carcinomas of the stomach, breast, and colon. Am J Clin Pathol 121: 884-892, 2004.

15. Nguyen MD, Plasil B, Wen $P$, Frankel WL. Mucin profiles in signet-ring cell carcinoma. Arch Pathol Lab Med 130: 799-804, 2006.

16. Kobayashi M, Fujinaga Y, Ota H. Reappraisal of the immunophenotype of pancreatic intraductal papillary mucinous neoplasms (IPMNs)-gastric pyloric and small intestinal immunophenotype expression in gastric and intestinal type IPMNs. Acta Histochem Cytochem 47: 45-57, 2014.

17. Sanada Y, Kunita S, Yoshida K. Comparison of histologic subtype and growth pattern in intraductal papillary-mucinous carcinoma of the pancreas. Oncol Rep 19: 1435-1443, 2008.

18. Koshita S, Noda Y, Ito K, et al. Pancreatic juice cytology with im- 
munohistochemistry to detect malignancy and histologic subtypes in patients with branch duct type intraductal papillary mucinous neoplasms of the pancreas. Gastrointest Endosc 85: 1036-1046, 2017.

19. Urata T. Localized type 1 autoimmune pancreatitis superimposed upon preexisting intraductal papillary mucinous neoplasms. World J Gastroenterol 19: 9127, 2013.

20. Koshita S, Noda Y, Ito K, et al. Branch duct intraductal papillary mucinous neoplasms of the pancreas involving type 1 localized autoimmune pancreatitis with normal serum IgG4 levels successfully diagnosed by endoscopic ultrasound-guided fine-needle aspiration and treated without pancreatic surgery. Intern Med 56: 1163-1167, 2017.

The Internal Medicine is an Open Access article distributed under the Creative Commons Attribution-NonCommercial-NoDerivatives 4.0 International License. To view the details of this license, please visit (https://creativecommons.org/licenses/ by-nc-nd/4.0/).

(C) 2018 The Japanese Society of Internal Medicine Intern Med 57: 1093-1099, 2018 